\title{
Basic or applied, it's the interaction that counts
}

\section{Toxicology must not be driven by politics: it's science that should drive decision-making.}

Sir - While we sympathize with some of the views expressed by Marcello Lotti and Pierluigi Nicotera in their Concepts essay "A risky business" (Nature 416, 481; 2002), the authors fail to acknowledge the wider role of toxicology in society. Toxicology is much more than the purely mechanistic studies they propose, and should not be incorporated into "the mainstream of fundamental biomedical research". Rather, it should draw from and interact with such research.

Toxicology has several functions: to identify the origin and nature of toxic insults to human health; to identify their mechanism of action; and to guide regulatory and public-health bodies in measures to remove or reduce toxicological threats. Although mechanistic studies are essential, it is just as important to know the nature of the compounds or environmental insults responsible for adverse effects, and the dosimetry of such exposures. This enables effective risk assessments and development of strategies to reduce risks to human health. Lotti and Nicotera fail to cite any example where mechanistic knowledge alone has allowed reliable risk predictions for humans.

Contrary to the authors' claim that basic research has become irrelevant to many toxicologists (partly justified in the past), recent national and international meetings demonstrate that toxicology has embraced and contributed to the advances of basic molecular and cell biology, as well as incorporating pharmacology, pathology, chemistry and epidemiology. Toxicology is a multidisciplinary subject, and there will always be situations which demand urgent action, irrespective of our understanding of basic biological mechanisms.

If the strategy proposed by Lotti and Nicotera had been applied to liver cancer in Southeast Asia and Africa, it would certainly have produced some interesting mechanistic data on hepatocarcinogenesis. But it was the integration of molecular dosimetry and epidemiological studies that identified aflatoxin B1 as a major risk factor for this cancer. Subsequently, the knowledge of carcinogenic mechanisms provided the rationale for measures to reduce the risk, such as improved food storage, and the design of chemoprevention trials. Similar remarks could be made about organophosphorus pesticides and neurotoxicity; polycyclic aromatic hydrocarbons, aromatic amines and carcinogenicity; accidental or deliberate chemical poisoning; the development of antidotes; and the relevance of endocrine disruptors to human health. In all these it is essential to carry out 'chemical-driven' as well as 'mechanism-driven' toxicology, using cutting-edge methodologies, to

The rapidly expanding knowledge about genetic polymorphisms of enzymes involved in activation (such as cytochrome P450) and detoxification (for example glutathione $S$-transferase and $\mathrm{N}$-acetyl transferase) of toxins shows that some population groups may be particularly susceptible to some chemicals. The mechanistic study of polymorphisms is valuable, but without considering the nature of the compounds to which individuals are exposed, such knowledge is of little practical value. The public is more concerned to know what compounds are toxic and to see them removed from the environment than to know in detail which biochemical mechanisms are occurring in their cells, important though that may be.

Society rightly demands assurances on generate meaningful risk assessments.

the safety of the environment, and this can be achieved only by the application of toxicological principles together with mechanistic understanding. Nevertheless, we should strive to ensure that political agendas do not drive toxicology, but rather that strong science should drive regulatory decisions. It is the interaction between fundamental and applied science that is important in toxicology, not one or the other component.

\section{Peter B. Farmer}

Biocentre, University of Leicester, University Road, Leicester LE1 7RH, UK

Other signatories to this letter:

Helmut Bartsch German Cancer Research Centre, Heidelberg Alan Boobis Imperial College, London, UK

Kevin Chipman School of Biosciences, University of Birmingham, UK Andy Gescher Department of Oncology, University of Leicester, UK Fred F. Kadlubar National Center for Toxicological Research, Jefferson, Arkansas, USA

Margaret M. Manson Biocentre, University of Leicester, UK David H. Phillips Institute of Cancer Research, London, UK David E. G. Shuker Department of Chemistry, Open University, UK James A. Swenberg University of North Carolina, USA

Steven R. Tannenbaum Massachusetts Institute of Technology, USA Christopher P. Wild University of Leeds, UK

\section{Fundamentals are still relevant in toxicology}

Sir-Marcello Lotti and Pierluigi Nicotera, in their Concepts essay on toxicology (Nature 416, 481; 2002), raise important issues about the seminal contribution made by the use of toxins as probes to our understanding of fundamental principles and functions of biological systems. But their claim that "basic research has, over the past two decades, become irrelevant to many toxicologists" is not true.

As the current and incoming presidents of the Society of Toxicology, a professional organization of more than 5,000 members, we can attest that our society has worked diligently to undo that outdated image of toxicology, and has taken up the challenge that "Toxicology research should urgently appraise its performance and join mainstream biomedical science". This is reflected in the quality of science presented at the society's meetings and published in leading journals. As part of a long-range plan to guide research, education and outreach (see www.toxicology.org), our priorities are to facilitate research with an emphasis on mechanisms of action, and to increase the use of relevant science in risk assessment and decision-making.

Lotti and Nicotera state "Toxicology is being shaped by worldwide political agendas, triggered by the public's desire for swift and precautionary solutions to the possible health effects of environmental chemicals." Risk assessment of chemical hazards is fraught with uncertainty, and the science of toxicology is sometimes misrepresented. It is in the public perception of hazards and health risks, and the blurred distinction between science and policy (see Roger A. Pielke's Commentary: Nature 416, 367-368; 2002) that toxicology receives a 'bad rap'.

Many factors go into risk-management decisions, and toxicology is but one piece of a complicated and highly political process managed by others with limited understanding of fundamental science. Basic research alone will not resolve uncertainty or conflict in risk assessment.

Fostering basic research is a primary goal of our discipline. But building better ways to put fundamental knowledge into risk assessment and regulatory practice is also vital. We believe that using knowledge to benefit human health is a responsibility shared by the entire biomedical research community. The National Institutes of Health and other sponsors of biomedical research continue to emphasize that the results obtained at the laboratory bench need to translate to improvements in the health of the public at large.

David L. Eaton ${ }^{\star}$, William F. Greenlee $\dagger$ ${ }^{\star}$ Center for Ecogenetics \& Environmental Health and School of Public Health, University of Washington, Seattle, Washington 98105-6099, USA $\dagger$ CIIT Centers for Health Research, PO Box 12137, Research Triangle Park, North Carolina 27709, USA 